\title{
Noncomplexed Cucurbituril-Mediated Structural Evolution of Layered Uranyl Terephthalate Compounds
}

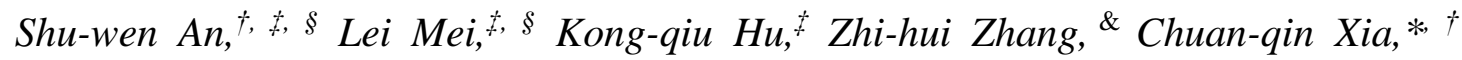
Zhi-fang Chai, $\dot{t}, \stackrel{\perp}{ }$ and Wei-qun Shi $* \dot{t}$

${ }^{\dagger}$ College of Chemistry, Sichuan University, Chengdu 610064, China

${ }^{*}$ Laboratory of Nuclear Energy Chemistry, Institute of High Energy Physics, Chinese Academy of Sciences, Beijing 100049, China

\& Jiangsu Key Laboratory of Advanced Catalytic Materials and Technology, Advanced Catalysis and Green Manufacturing Collaborative Innovation Center, Changzhou University, 213164 Changzhou, China

${ }^{\perp}$ Engineering Laboratory of Advanced Energy Materials, Ningbo Institute of Industrial Technology, Chinese Academy of Sciences, Ningbo 315201, China 


\section{Table of Content}

\section{S1 General Methods}

\section{S2 Typical Figures}

Figure S1. Asymmetric unit of compound 2.

Figure S2. PXRD patterns of cpmpound 2 before $\mathrm{Cs}^{+}$sorption and after $\mathrm{Cs}^{+}$sorption.

Figure S3. The four kinds of coordination modes of $\mathbf{T P}^{2-}$ ligand in this work.

Figure S4. Simulated and experimental PXRD patterns of as-synthesized compound 2.

Figure S5. Simulated and experimental PXRD patterns of as-synthesized compound $\mathbf{3}$.

Figure S6. TGA results for compound $\mathbf{2}$ and $\mathbf{3}$.

Figure S7. FTIR spectra of compound $\mathbf{2}$ and $\mathbf{3}$.

\section{S3 Typical Tables}

Table S1. Selected bond lengths related to the uranyl centers in compounds 1-6.

Table S2. $\mathrm{Cs}^{+}$exchange measurements using compound $\mathbf{2}$. 


\section{S1 General Methods}

$\mathrm{Cs}^{+}$exchange experiments. $\mathrm{Cs}^{+}$exchange measurements of compound $\mathbf{2}$ were studied by soaking $4 \mathrm{mg}$ compound 2 in $20 \mathrm{~mL}$ of water solutions of $\mathrm{CsNO}_{3}(1-200 \mathrm{ppm})$ with $\mathrm{V} / \mathrm{m}$ ratio of 5000 $\mathrm{mL} / \mathrm{g}$ ( $\mathrm{V}$ is the volume of solutions and $\mathrm{m}$ is the mass of the adsorbent), the mixture was put on a shaker for two days, and the ICP-MS measurements were used to detect the concentration of final $\mathrm{Cs}^{+}$solutions.

\section{S2 Typical Figures}

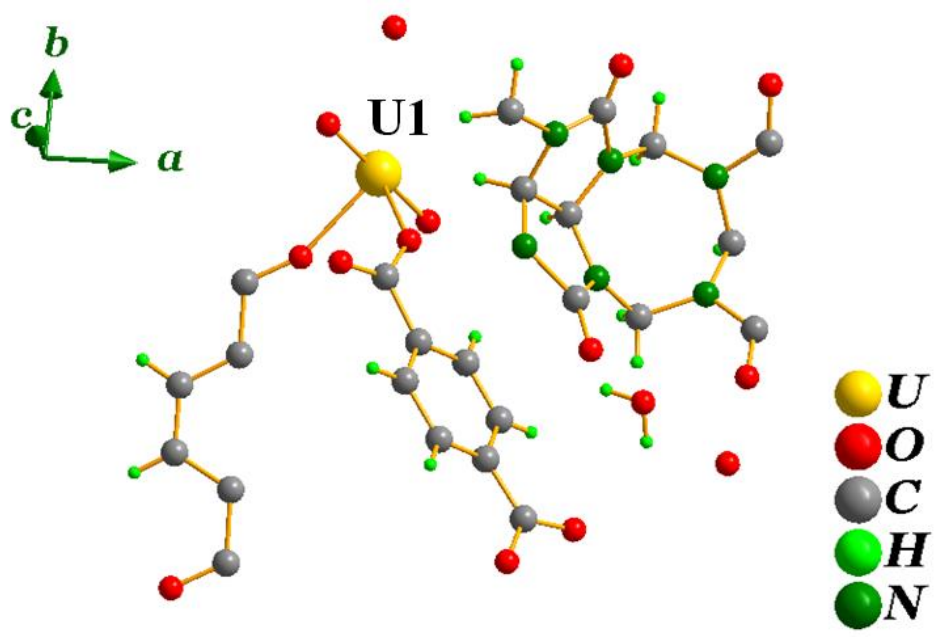

Figure S1. Asymmetric unit of compound 2.
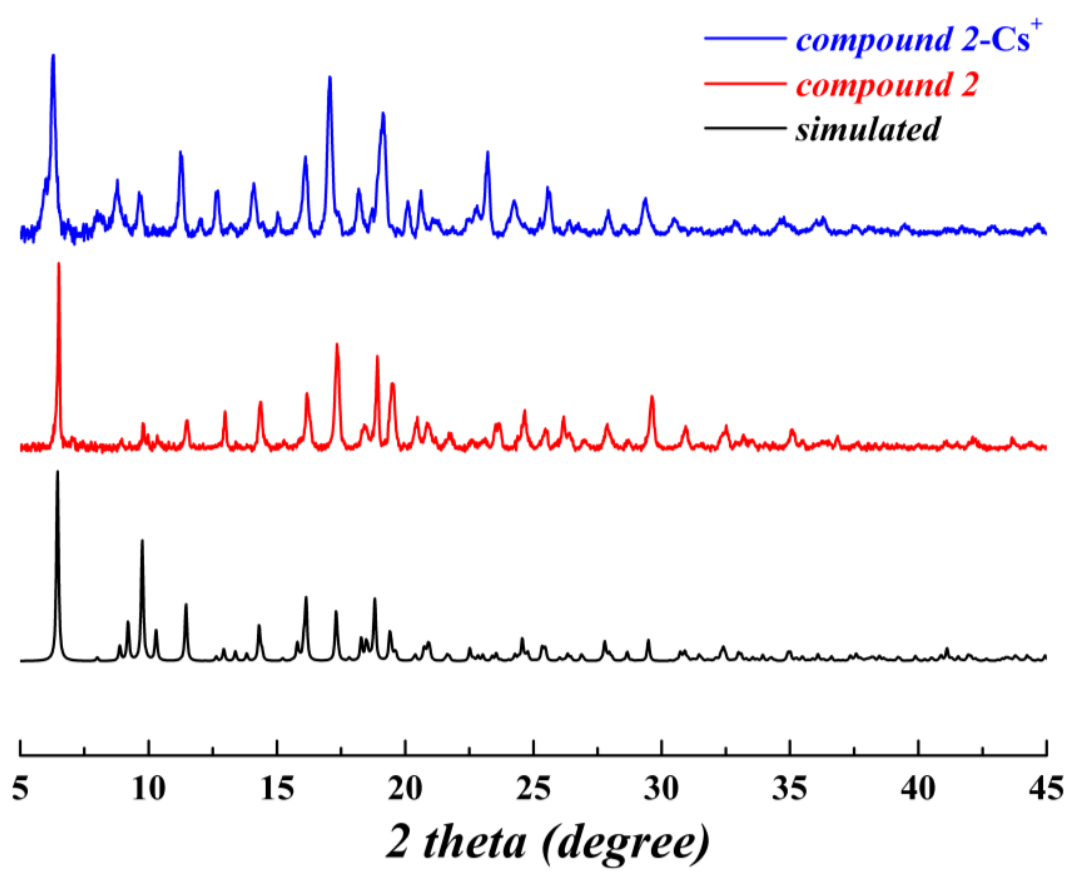

Figure S2. PXRD patterns of cpmpound 2 before $\mathrm{Cs}^{+}$sorption and after $\mathrm{Cs}^{+}$sorption. 


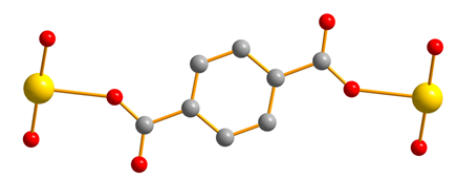

$\mu_{2}-\eta^{1}: \eta^{1}$

mode I

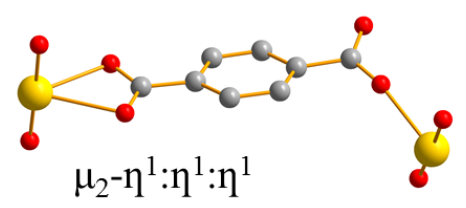
mode III

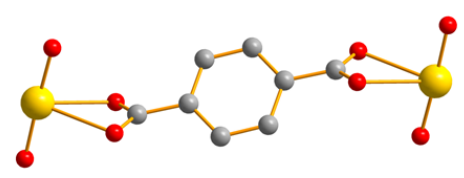

$$
\mu_{2}-\eta^{1}: \eta^{1}: \eta^{1}: \eta^{1}
$$
mode II

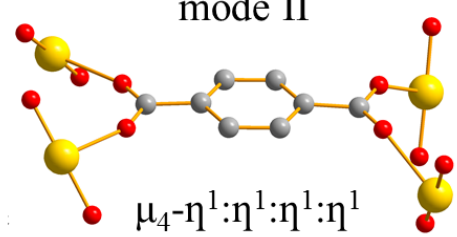
mode IV

Figure S3. The four kinds of coordination modes of $\mathbf{T P}^{2-}$ ligand in this work.

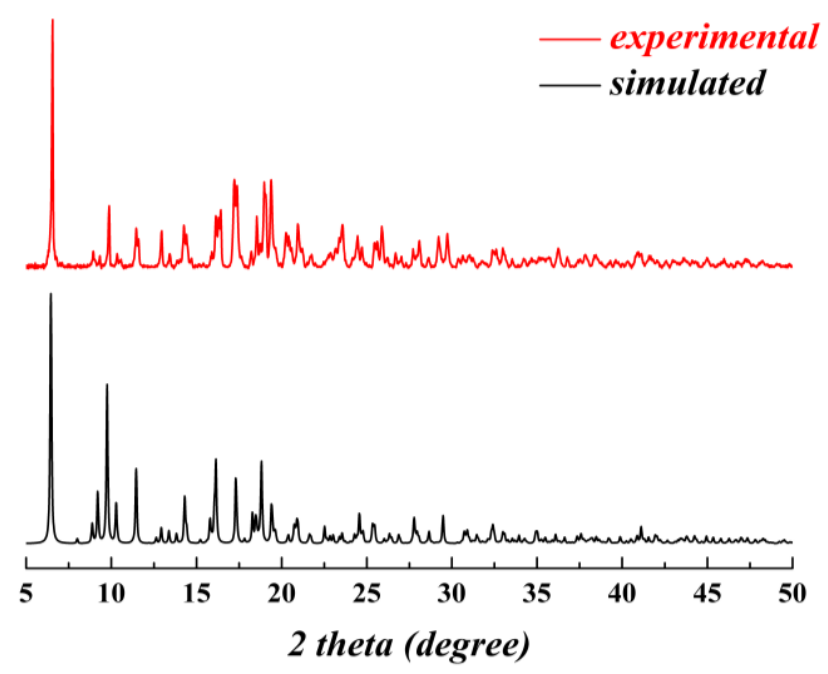

Figure S4. Simulated and experimental PXRD patterns of as-synthesized compound 2.
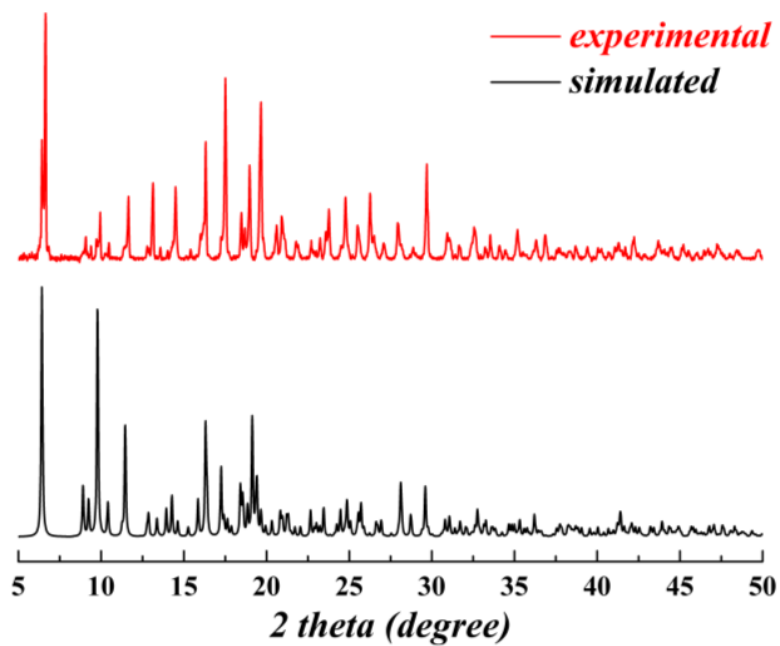

Figure S5. Simulated and experimental PXRD patterns of as-synthesized compound 2. 


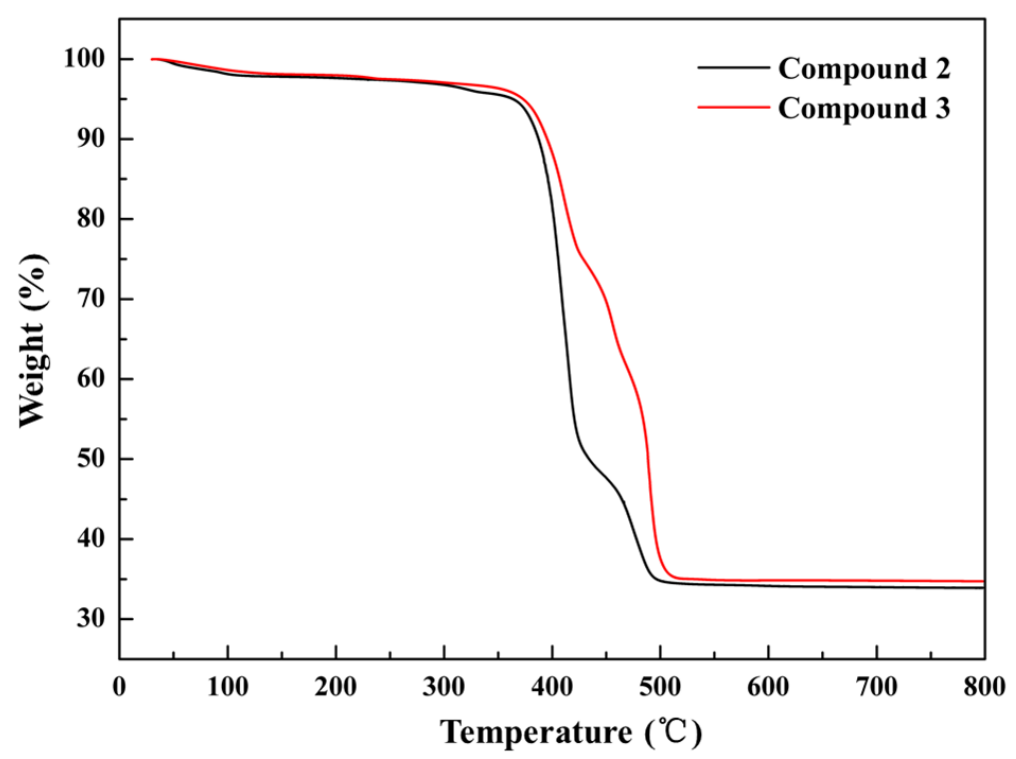

Figure S6. TGA results for compound 2 and 3.

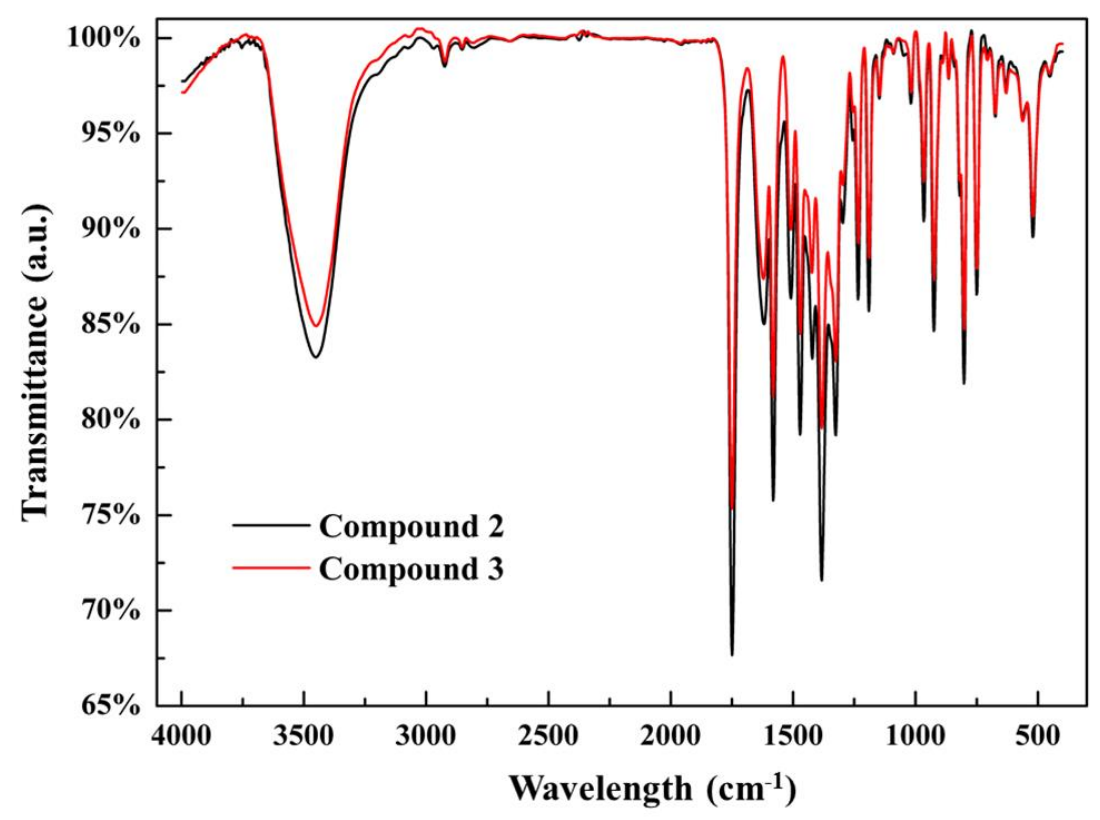

Figure S7. FTIR spectra of compound 2 and $\mathbf{3}$.

\section{S3 Typical Tables}

Table S1. Selected bond lengths related to the uranyl centers in compounds 1-6.

\begin{tabular}{llll}
\hline \multicolumn{3}{c}{ Compound 1 } \\
\hline $\mathrm{U} 1-\mathrm{O} 13$ & $2.259(3)$ & $\mathrm{C} 35-\mathrm{H} 35 \mathrm{~B} \cdots \mathrm{O} 18$ & $2.7938(1)$ \\
$\mathrm{U} 1-\mathrm{O} 17$ & $2.360(3)$ & $\mathrm{C} 36-\mathrm{H} 36 \mathrm{~A} \cdots \mathrm{O} 18$ & $2.5525(1)$ \\
$\mathrm{U} 1-\mathrm{O} 18$ & $1.781(3)$ & $\mathrm{C} 4-\mathrm{H} 4 \cdots \mathrm{O} 10$ & $2.9169(1)$ \\
$\mathrm{U} 1-\mathrm{O} 19$ & $1.781(3)$ & $\mathrm{C} 6-\mathrm{H} 6 \cdots \mathrm{O} 11$ & $2.8080(1)$ \\
$\mathrm{U} 1-\mathrm{O} 20$ & $2.494(3)$ & $\mathrm{C} 17-\mathrm{H} 17 \mathrm{~B} \cdots \mathrm{O} 12$ & $2.4207(1)$ \\
$\mathrm{U} 1-\mathrm{O} 21$ & $2.464(3)$ & $\mathrm{C} 19-\mathrm{H} 19 \cdots \mathrm{O} 12$ & $2.4757(1)$ \\
\hline
\end{tabular}




\begin{tabular}{|c|c|c|c|}
\hline \multirow[t]{4}{*}{ U1-O15 } & $2.342(3)$ & $\mathrm{C} 22-\mathrm{H} 22 \cdots \mathrm{O} 15$ & $2.7482(1)$ \\
\hline & & $\mathrm{C} 22-\mathrm{H} 22 \cdots \mathrm{O} 14$ & $2.4567(1)$ \\
\hline & & $\mathrm{C} 24-\mathrm{H} 24 \mathrm{~B} \cdots \mathrm{O} 14$ & $2.6857(1)$ \\
\hline & & $\mathrm{C} 23-\mathrm{H} 23 \mathrm{~A} \cdots \mathrm{O} 14$ & $2.3380(1)$ \\
\hline \multicolumn{4}{|c|}{ Compound 2} \\
\hline U1-O1 & $1.7764(85)$ & $\mathrm{C} 16-\mathrm{H} 16 \cdots \mathrm{O} 8$ & $2.4751(66)$ \\
\hline $\mathrm{U} 1-\mathrm{O} 2$ & $1.7510(83)$ & $\mathrm{C} 16-\mathrm{H} 16 \cdots \mathrm{O} 7$ & $2.5201(82)$ \\
\hline U1-O5 & $2.4138(79)$ & $\mathrm{C} 12-\mathrm{H} 12 \cdots \mathrm{O} 5$ & 2.5304(97) \\
\hline U1-O6 & $2.4732(84)$ & $\mathrm{C} 22-\mathrm{H} 22 \cdots \mathrm{O} 3$ & 2.6416(99) \\
\hline U1-O8 & $2.3371(80)$ & C6-H6 ..O12 & $2.6638(173)$ \\
\hline $\mathrm{U} 1-\mathrm{O} 3$ & $2.2982(87)$ & $\mathrm{C} 18-\mathrm{H} 18 \mathrm{~B} \cdots \mathrm{O} 1$ & $2.7284(84)$ \\
\hline \multirow[t]{2}{*}{ U1-O7 } & $2.3404(81)$ & $\mathrm{C} 16-\mathrm{H} 16 \cdots \mathrm{O} 1$ & $2.7684(86)$ \\
\hline & & $\mathrm{C} 23-\mathrm{H} 23 \cdots \mathrm{O} 3$ & $2.8461(86)$ \\
\hline
\end{tabular}

\section{Compound 3}

\begin{tabular}{llll|}
\hline $\mathrm{U} 1-\mathrm{O} 2$ & $2.292(7)$ & $\mathrm{C} 7-\mathrm{H} 7 \cdots \mathrm{O} 15$ & $2.7583(1)$ \\
\hline $\mathrm{U} 1-\mathrm{O} 5$ & $1.744(6)$ & $\mathrm{C} 21-\mathrm{H} 21 \mathrm{~B} \cdots \mathrm{O} 6$ & $2.7899(1)$ \\
\hline $\mathrm{U} 1-\mathrm{O} 6$ & $1.768(6)$ & $\mathrm{C} 21-\mathrm{H} 21 \mathrm{~B} \cdots \mathrm{O} 7$ & $2.8020(1)$ \\
\hline $\mathrm{U} 1-\mathrm{O} 7$ & $2.328(6)$ & $\mathrm{C} 22-\mathrm{H} 22 \cdots \mathrm{O} 6$ & $2.8156(1)$ \\
$\mathrm{U} 1-\mathrm{O} 3$ & $2.408(8)$ & $\mathrm{C} 22-\mathrm{H} 22 \cdots \mathrm{O} 7$ & $2.4731(1)$ \\
\hline $\mathrm{U} 1-\mathrm{O} 4$ & $2.479(7)$ & $\mathrm{C} 22-\mathrm{H} 22 \cdots \mathrm{O} 8$ & $2.5201(1)$ \\
\hline $\mathrm{U} 1-\mathrm{O} 8$ & $2.341(6)$ & & \\
\hline
\end{tabular}

\section{Compound 4}

\begin{tabular}{|llll|}
\hline U1-O2 & $1.7756(1)$ & $\mathrm{C} 38-\mathrm{H} 38 \cdots \mathrm{O} 11$ & $2.229(5)$ \\
\hline U1-O1 & $1.7636(1)$ & $\mathrm{C} 56-\mathrm{H} 56 \cdots \mathrm{O} 8$ & $2.363(5)$ \\
\hline U1-O6 & $2.4788(1)$ & $\mathrm{C} 56-\mathrm{H} 56 \cdots \mathrm{O} 16$ & $2.463(5)$ \\
\hline U1-O5 & $2.4459(1)$ & $\mathrm{C} 42-\mathrm{H} 42 \mathrm{~B} \cdots \mathrm{O} 4$ & $2.510(5)$ \\
\hline U1-O15 & $2.4814(1)$ & $\mathrm{C} 39-\mathrm{H} 39 \cdots \mathrm{O} 4$ & $2.531(5)$ \\
\hline U1-O16 & $2.4492(1)$ & $\mathrm{C} 54-\mathrm{H} 54 \mathrm{~B} \cdots \mathrm{O} 16$ & $2.558(5)$ \\
\hline U1-O8 & $2.4227(1)$ & $\mathrm{C} 38-\mathrm{H} 38 \cdots \mathrm{O} 9$ & $2.587(5)$ \\
\hline U1-O7 & $2.4688(1)$ & $\mathrm{C} 57-\mathrm{H} 57 \cdots \mathrm{O} 2$ & $2.589(5)$ \\
\hline U2-O4 & $1.7532(1)$ & $\mathrm{C} 53-\mathrm{H} 53 \mathrm{~B} \cdots \mathrm{O} 8$ & $2.657(5)$ \\
\hline U2-O3 & $1.7810(1)$ & $\mathrm{C} 36-\mathrm{H} 36 \mathrm{~A} \cdots \mathrm{O} 3$ & $2.662(5)$ \\
\hline U2-O10 & $2.4829(1)$ & $\mathrm{O} 4-\mathrm{O} 4 \cdots \mathrm{H} 11$ & $2.724(5)$ \\
\hline U2-O9 & $2.4747(1)$ & $\mathrm{O} 5-\mathrm{O} 5 \cdots \mathrm{H} 60 \mathrm{~B}$ & $2.797(5)$ \\
\hline U2-O12 & $2.4760(1)$ & $\mathrm{C} 41-\mathrm{H} 41 \mathrm{~B} \cdots \mathrm{O} 4$ & $2.807(5)$ \\
\hline U2-O13 & $2.4844(1)$ & $\mathrm{C} 38-\mathrm{H} 38 \cdots \mathrm{O} 3$ & $2.936(5)$ \\
\hline U2-O14 & $2.4346(1)$ & & \\
\hline U2-O11 & $2.4011(1)$ & & \\
\hline
\end{tabular}

\section{Compound 5}




\begin{tabular}{llll}
\hline $\mathrm{U} 1-\mathrm{O} 003$ & $2.304(3)$ & $\mathrm{C} 34-\mathrm{H} 34 \mathrm{~A} \cdots \mathrm{O} 10$ & $2.638(5)$ \\
$\mathrm{U} 1-\mathrm{O} 005$ & $2.310(4)$ & $\mathrm{C} 31-\mathrm{H} 31 \mathrm{~B} \cdots \mathrm{O} 1$ & $2.65(5)$ \\
$\mathrm{U} 1-\mathrm{O} 9$ & $1.782(5)$ & $\mathrm{C} 20-\mathrm{H} 20 \mathrm{~B} \cdots \mathrm{O} 2$ & $2.669(5)$ \\
$\mathrm{U} 1-\mathrm{O} 10$ & $1.746(6)$ & $\mathrm{C} 19-\mathrm{H} 19 \mathrm{~B} \cdots \mathrm{O} 30$ & $2.702(4)$ \\
$\mathrm{U} 1-\mathrm{O} 28$ & $2.297(4)$ & $\mathrm{C} 7-\mathrm{H} 7 \mathrm{~B} \cdots \mathrm{O} 10$ & $2.706(5)$ \\
\hline $\mathrm{U} 1-\mathrm{O} 29$ & $2.482(4)$ & $\mathrm{C} 59-\mathrm{H} 59 \cdots \mathrm{O} 21$ & $2.775(3)$ \\
$\mathrm{U} 1-\mathrm{O} 30$ & $2.512(3)$ & $\mathrm{C} 7-\mathrm{H} 7 \mathrm{~A} \cdots \mathrm{O} 24$ & $2.914(3)$ \\
& & $\mathrm{C} 7-\mathrm{H} 7 \mathrm{~B} \cdots \mathrm{O} 003$ & $2.953(4)$ \\
& & $\mathrm{C} 22-\mathrm{H} 22 \cdots \mathrm{O} 2$ & $2.983(6)$ \\
& & $\mathrm{C} 42-\mathrm{H} 42 \cdots \mathrm{O} 29$ & $2.282(5)$ \\
& & $\mathrm{C} 5-\mathrm{H} 5 \cdots \mathrm{O} 003$ & $2.323(3)$ \\
& & $\mathrm{C} 22-\mathrm{H} 22 \cdots \mathrm{O} 9$ & $2.381(4)$ \\
& & $\mathrm{C} 19-\mathrm{H} 19 \mathrm{~B} \cdots \mathrm{O} 9$ & $2.448(3)$ \\
& & $\mathrm{C} 30-\mathrm{H} 30 \cdots \mathrm{O} 1$ & $2.493(6)$ \\
\hline
\end{tabular}

\begin{tabular}{|c|c|c|c|}
\hline \multicolumn{4}{|c|}{ Compound 6} \\
\hline $\mathrm{U} 1-\mathrm{O} 2$ & $1.7748(10)$ & C102-H102‥O30 & $2.2508(11)$ \\
\hline U1-O6 & $2.4750(13)$ & $\mathrm{C} 54-\mathrm{H} 54 \cdots \mathrm{O} 35$ & $2.2820(11)$ \\
\hline U1-O16 & $2.4618(14)$ & C69-H69A … & $2.3115(11)$ \\
\hline U1-O15 & $2.4806(14)$ & $\mathrm{C} 86-\mathrm{H} 86 \cdots \mathrm{O} 26$ & $2.3218(15)$ \\
\hline $\mathrm{U} 1-\mathrm{O} 1$ & $1.7537(10)$ & 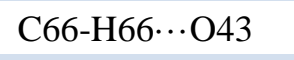 & $2.3384(12)$ \\
\hline U1-O4 & $2.4408(15)$ & C87-H87A $\cdots$ O 26 & $2.3411(11)$ \\
\hline U1-O5 & $2.4678(15)$ & $\mathrm{C} 77-\mathrm{H} 77 \cdots \mathrm{O} 45$ & $2.3600(14)$ \\
\hline U1-O3 & $2.4626(12)$ & C91-H91A $\cdots$ O28 & $2.3718(11)$ \\
\hline $\mathrm{U} 2-\mathrm{O} 14$ & $2.4827(15)$ & C37-H37B $\cdots$ O35 & $2.3837(12)$ \\
\hline $\mathrm{U} 2-\mathrm{O} 8$ & $2.4830(15)$ & $\mathrm{C} 72-\mathrm{H} 72 \cdots \mathrm{O} 44$ & $2.3990(11)$ \\
\hline $\mathrm{U} 2-\mathrm{O} 12$ & $2.4527(15)$ & C38-H38A ‥ O37 & $2.4115(14)$ \\
\hline U2-O9 & $1.7746(10)$ & C75-H75A $\cdots \mathrm{O} 45$ & $2.4135(10)$ \\
\hline U2-O11 & $2.4659(13)$ & $\mathrm{C} 93-\mathrm{H} 93 \cdots \mathrm{O} 28$ & $2.4234(16)$ \\
\hline $\mathrm{U} 2-\mathrm{O} 13$ & $2.4697(14)$ & $\mathrm{C} 89-\mathrm{H} 89 \cdots \mathrm{O} 27$ & $2.4689(13)$ \\
\hline $\mathrm{U} 2-\mathrm{O} 7$ & $2.4715(13)$ & $\mathrm{C} 56-\mathrm{H} 56 \cdots \mathrm{O} 33$ & $2.4770(14)$ \\
\hline $\mathrm{U} 2-\mathrm{O} 10$ & $1.7846(11)$ & $\mathrm{C} 35-\mathrm{H} 35 \cdots \mathrm{O} 36$ & $2.4894(12)$ \\
\hline U3-O19 & $2.4318(15)$ & $\mathrm{C} 41-\mathrm{H} 41 \cdots \mathrm{O} 37$ & $2.5309(10)$ \\
\hline U3-O17 & $1.7893(9)$ & C74-H74A $\cdots$ O39 & $2.5973(16)$ \\
\hline $\mathrm{U} 3-\mathrm{O} 20$ & $2.3862(13)$ & C39-H39B $\cdots$ O37 & $2.6024(17)$ \\
\hline \multirow[t]{8}{*}{ U3-O18 } & $2.4876(12)$ & C39-H39A ‥ O47 & $2.7471(13)$ \\
\hline & & C74-H74B $\cdots$ O45 & $2.8555(15)$ \\
\hline & & $\mathrm{C} 35-\mathrm{H} 35 \cdots \mathrm{O} 37$ & $2.8938(14)$ \\
\hline & & C39-H39A $\cdots$ O31 & $2.9125(16)$ \\
\hline & & 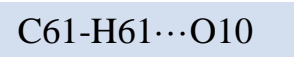 & $2.5460(12)$ \\
\hline & & C61-H61O12 & $2.7093(16)$ \\
\hline & & C97-H97B $\cdots \mathrm{O} 13$ & $2.4933(13)$ \\
\hline & & C50-H50B $\cdots \mathrm{O} 21$ & $2.5889(13)$ \\
\hline
\end{tabular}


Table S2. $\mathrm{Cs}^{+}$exchange measurements using compound 2 .

\begin{tabular}{cccccc}
\hline Initial Cs concentration (ppm) & $\mathbf{1}$ & $\mathbf{1 0}$ & $\mathbf{2 0}$ & $\mathbf{1 0 0}$ & $\mathbf{2 0 0}$ \\
\hline $\mathbf{V} / \mathbf{m}(\mathbf{m L} / \mathbf{g})$ & 5000 & 5000 & 5000 & 5000 & 5000 \\
\hline Final concentration (ppm) & 0.70 & 6.79 & 13.68 & 91.70 & 190.86 \\
\% Cs Removal & 30 & 32.1 & 31.6 & 8.3 & 4.57 \\
\hline Adsorption capacity (mg/g) & 1.5 & 16.05 & 31.6 & 41.5 & 45.7 \\
\hline
\end{tabular}

